\title{
Monitoramento da Integridade de Estruturas Aeronáuticas Utilizando um Método Inteligente Baseado em Sistemas Imunológicos Artificiais
}

\author{
$\underline{\text { Fernando P. A. Lima }}^{\dagger} \quad$ Adriano. S. Souza ${ }^{\dagger} \quad$ Simone. S. F. Souza ${ }^{\dagger}$ \\ Mara. L. M. Lopes ${ }^{*} \quad$ Fábio R. Chavarette ${ }^{\dagger}$ \\ ${ }^{\dagger}$ Departamento de Engenharia Mecânica, Faculdade de Engenharia de Ilha Solteira (FEIS) \\ *Departamento de Matemática, Faculdade de Engenharia de Ilha Solteira (FEIS) \\ ‘Departamento de Engenharia Elétrica, Faculdade de Engenharia de Ilha Solteira (FEIS) \\ Universidade Estadual Paulista "Júlio de Mesquita Filho" (UNESP), Ilha Solteira, SP, BRASIL \\ E-mails: engfernandoparra@gmail.com, eng.adrianosouza@gmail.com, \\ simonefrutuoso.mat@gmail.com,mara@mat.feis.unesp.br, fabioch@mat.feis.unesp.br
}

Palavras-chaves: Monitoramento da Integridade, Estruturas Aeronáuticas, Sistemas Imunológicos Artificiais, Algoritmo de Seleção Negativa.

Resumo: Neste artigo apresenta-se um método inteligente baseado nos sistemas imunológicos artificiais para monitoramento da integridade de estruturas aeronáuticas. Desta forma, tomando-se como base um processo biológico do sistema imunológico, utiliza-se o Algoritmo de Seleção Negativa para identificar e caracterizar amostras de falhas estruturais. A principal aplicação deste método é auxiliar profissionais a inspecionar estruturas aeronáuticas, detectar e caracterizar falhas, bem como a tomada de decisões, visando evitar catástrofes. Para avaliar este método, foi realizada a modelagem e simulação de sinais a partir de um modelo genérico utilizando uma viga de alumínio. Os resultados obtidos pelo método são excelentes, apresentando robustez e precisão.

\section{Introdução}

Há alguns anos a linha de pesquisa de monitoramento da integridade de estruturas aeronáuticas cresceu se tornando muito importante do ponto de vista econômico e principalmente de segurança. De uma forma genérica, um Sistema de Monitoramento da Integridade de Estruturas (SMIE) deve ser capaz de executar tarefas como: aquisição e processamento de dados, validação e análise, de forma a identificar e interpretar mudanças adversas em uma estrutura, visando facilitar tomada de decisões e garantir segurança [8].

As falhas estruturais podem ocorrer devido a diversos fatores tais como: desgaste de um componente, afrouxamento de uniões aparafusadas, trincas ou mesmo a combinação destes elementos. Independente da origem, na grande maioria dos casos, falha estrutural provoca uma sensível variação dos parâmetros espaciais da estrutura tais como: redução da rigidez estrutural, ligeira redução da massa e um aumento do amortecimento, o que leva a uma mudança do comportamento dinâmico da estrutura. Assim, a variação dos parâmetros espaciais afeta os principais parâmetros dinâmicos, funções de respostas, frequências de ressonância, razão de amortecimento e modos próprios da estrutura [13].

Toda estrutura antes de ser colocada em uso geralmente passa por uma avaliação e testes de integridade. Neste caso uma avaliação não-destrutiva (NDE) é realizada com o objetivo de estimar o grau de segurança e confiabilidade da estrutura. As tradicionais técnicas de avaliação NDE (inspeção ultrassônica, radiografia-X, testes de emissão acústica, etc.) podem possuir um bom aparato instrumental e serem bem formuladas para diversas aplicações, no entanto, estas técnicas não podem satisfazer necessidades crescentes no contexto do monitoramento da integridade de estruturas, quando as mesmas estão em uso [6].

Desta forma, os SMIE veem sofrendo uma evolução natural, devido ao avanço de tecnologias de sensores, materiais inteligentes, processamento de dados e sinais, bem como a crescente demanda de estruturas que necessitem de avaliações em tempo real, como aviões. 
Neste contexto, a utilização de sensores inteligentes em conjunto com técnicas inteligentes para análise e tomada de decisões, se tornam possíveis alternativas para os SMIE.

$\mathrm{Na}$ literatura existem várias propostas de trabalhos utilizando materiais e sistemas inteligentes em SMIE, os quais apresentam bom desempenho e precisão. Na sequência apresentam-se os trabalhos mais relevantes.

Em [9] apresentaram a aplicação de um algoritmo genético em conjunto com uma rede neural para detecção e localização de falhas em um modelo numérico de uma viga. No trabalho [13] os autores utilizaram uma rede multicamadas com treinamento via backpropagation para predição de falhas em vigas CFRP (reforçadas com fibra de carbono). Em [7] utilizou-se o método da impedância eletromecânica para monitorar estruturas aeroespaciais com sensores piezelétricos ativos acoplados. No artigo de [12] os autores combinaram a técnica de impedância elétrica com um algoritmo PSO (Particle Swarm Optimization) para diagnosticar falhas. Em [2] propõe-se um sistema fuzzy para detecção de falhas estruturais usando curvaturas das formas modais. Em [14] foi proposto uma algoritmo genético multinodal para diagnosticar danos em uma ponte de treliça de aço.

Neste artigo, apresenta-se um SMIE baseado nos sistemas imunológicos artificiais para monitorar a integridade de estruturas aeronáuticas. A partir da aquisição e processamento dos sinais, aplica-se o algoritmo de seleção negativa (ASN) [5] para identificar e caracterizar as falhas. Para avaliar este método, utilizou-se uma base de dados de sinais simulados a partir de um modelo numérico de uma viga de alumínio, que neste caso representa uma estrutura aeronáutica. A viga foi modelada através da técnica de elementos finitos e simulada em Matlab [11]. Neste trabalho escolheu-se por empregar os sistemas imunológicos artificiais (SIA) devido as suas características naturais de aprendizagem e reconhecimento de padrões e por apresentarem bom desempenho e eficiência em outros tipos de problemas, conforme destacado em [10].

Este artigo está organizado da seguinte forma: A descrição do algoritmo de seleção negativa (ASN) está na seção 2. Na seção 3 apresentam-se a modelagem e simulações realizadas. Na seção 4 apresenta-se a metodologia proposta. As aplicações e resultados são apresentados na seção 5 e por fim, a seção 6 apresenta a conclusão para este trabalho.

\section{Algoritmo de Seleção Negativa}

O algoritmo de seleção negativa (ASN) foi proposto por [6] para detecção de mudanças em estados de sistemas, e é baseado na seleção negativa de linfócitos $\mathrm{T}$ dentro do timo. Este processo trabalha com a discriminação de células próprias e não-próprias. $\mathrm{O}$ algoritmo é executado em duas fases, conforme ilustrado nos fluxogramas apresentados na Figura 1 [4]:

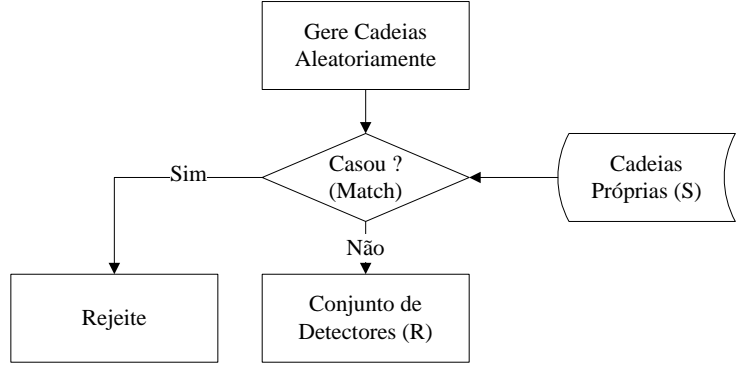

(a)

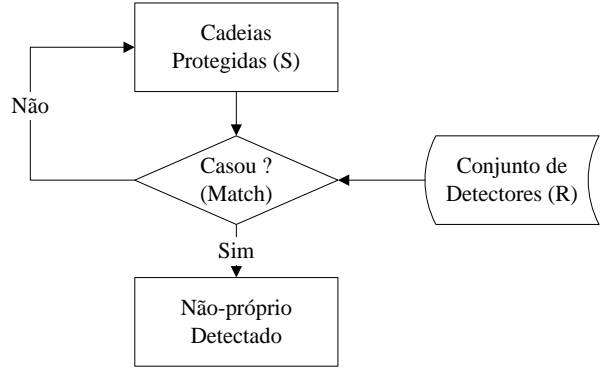

(b)

Figura 1: Fluxogramas do ASN.

A fase de censoriamento do ASN consiste-se basicamente em gerar um conjunto de detectores. Os detectores são análogos às células tipo $\mathrm{T}$, maturadas capazes de reconhecer agentes patogênicos. A fase de monitoramento consiste-se em monitorar um sistema, visando identificar uma mudança no comportamento do mesmo, e assim classificar esta mudança utilizando o conjunto de detectores criados na fase de censoriamento [3]. 


\subsection{Critério de Afinidade}

Para avaliar a afinidade entre as cadeias e afirmar que são semelhantes, utiliza-se um critério conhecido como casamento. O casamento pode ser perfeito ou parcial [10]. Neste trabalho utiliza-se o casamento parcial. No casamento parcial, uma quantidade de pontos entre os padrões deve ter o mesmo valor para se confirmar o casamento, sendo a quantidade (taxa de afinidade) definida previamente. A taxa de afinidade representa o grau de semelhança necessário para ocorrer o casamento entre os dois padrões. A taxa de afinidade é definida através da seguinte relação [1]:

$$
T A f=\left(\frac{A n}{A t}\right) * 100
$$

sendo:

TAf : taxa de afinidade;

An : número de cadeias normais no problema (cadeias próprias);

At : número total de cadeias no problema (cadeias próprias e não-próprias).

A equação (1) propõe uma relação estatística entre todas as amostras do problema para o cálculo da taxa de afinidade. A expressão (2) representa a forma de quantificar a afinidade total entre os padrões [1]:

$$
A f_{T}=\frac{\sum_{i=1}^{L} V c}{L} * 100
$$

sendo:

$A f_{T}: \%$ de afinidade entre os padrões analisados;

$L \quad$ : quantidade total de variáveis;

Vc : variáveis casada;

$\sum_{i=1}^{L} V c:$ somatória (quantidade) de variáveis casadas.

Desta forma se $A f_{T}$ for maior ou igual a $T A f$ ocorre o casamento entre os dois padrões (são considerados semelhantes).

\section{Modelagem e Simulação}

Para avaliar a metodologia proposta neste trabalho, foi utilizado o método de elementos finitos para modelar uma viga de alumínio na condição engastada-livre discretizada com 10 elementos finitos com 2 graus de liberdade cada. As propriedades do material empregado são: módulo de elasticidade $\mathrm{E}=700 \mathrm{GPa}$, densidade em massa $\gamma=2710 \mathrm{~kg} / \mathrm{m}^{3}$. As dimensões são: $500 \mathrm{~mm}$ de comprimento, $25 \mathrm{~mm}$ de largura e $5 \mathrm{~mm}$ de espessura. A figura 2 ilustra a viga modelada.

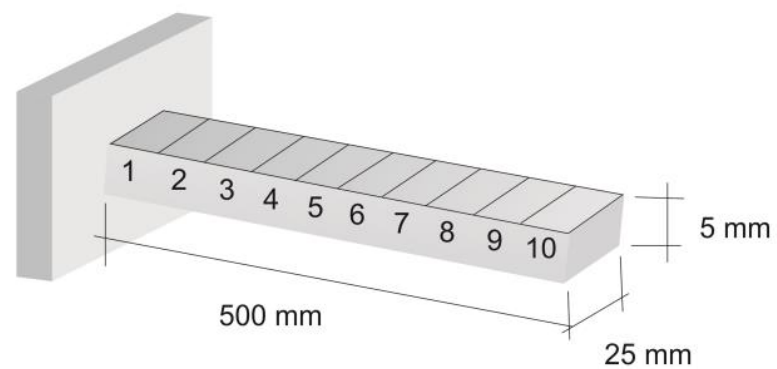

Figura 2: Viga simulada.

Através da viga modelada pela técnica de elementos finitos foram realizadas diversas simulações com diferentes porcentagens de desgastes e localizações das falhas. A base de dados 
que foi gerada é constituída de sinais capturados por um acelerômetro fixado na viga. Em todas as simulações a viga foi excitada no $3^{\circ}$ grau de liberdade e o sinal foi capturado no $19^{\circ}$ grau de liberdade. Desta forma, foram simulados 1500 sinais na estrutura, sendo 400 sinais sem desgaste (condição base-line) e 1400 sinais com desgastes (falhas estruturais). Os sinais em falha foram simulados com níveis de desgaste de $1,5,10,15,20,25,30,35,40,45$ e $50 \%$ e para cada nível de desgaste a falha foi posicionada em todos os elementos finitos. Na Tabela 1 (a) apresenta-se a quantidade de simulações para cada nível de falha. Na Tabela 1 (b) apresentase a quantidade de simulações para cada localização da falha em cada um dos níveis de falha, isto é, para cada nível de falha foram gerados 10 simulações em cada localização.

Tabela 1 - Configurações das simulações.

\begin{tabular}{c|c}
\hline Nível de Falha & $\begin{array}{c}\text { Quantidade de } \\
\text { simulações }\end{array}$ \\
\hline $0 \%$ & 400 \\
$1 \%$ & 100 \\
$5 \%$ & 100 \\
$10 \%$ & 100 \\
$15 \%$ & 100 \\
$20 \%$ & 100 \\
$25 \%$ & 100 \\
$30 \%$ & 100 \\
$35 \%$ & 100 \\
$40 \%$ & 100 \\
$45 \%$ & 100 \\
$50 \%$ & 100 \\
\hline Total & 1500 \\
\hline
\end{tabular}

\begin{tabular}{c|c}
\hline Localização & $\begin{array}{c}\text { Quantidade de } \\
\text { simulações }\end{array}$ \\
\hline 1 & 10 \\
2 & 10 \\
3 & 10 \\
4 & 10 \\
5 & 10 \\
6 & 10 \\
7 & 10 \\
8 & 10 \\
9 & 10 \\
10 & 10 \\
\hline Total & 100 \\
\hline
\end{tabular}

(a)

(b)

\section{Metodologia Proposta}

O sistema de monitoramento da integridade de estruturas aeronáuticas apresentado nesta seção é baseado nos sistemas imunológicos artificiais [4], em especial no algoritmo de seleção negativa [5]. O método proposto se divide em três módulos: aquisição de dados, censoriamento e o monitoramento dos dados.

Na sequência apresenta-se o sistema de monitoramento da integridade estrutural.

\subsection{Sistema de Monitoramento da Integridade Estrutural}

O SMIE é composto por três módulos principais, sendo a aquisição de dados, o censoriamento e o monitoramento.

O módulo de aquisição de dados é composto pelo aparato experimental para capturar os sinais na estrutura, como: sensores, atuadores, acelerômetros, etc.

O módulo de censoriamento é realizado de forma off-line e serve para gerar o conjunto de detectores de falhas que será utilizado no monitoramento dos dados. Neste módulo o conjunto de detectores de falhas é composto por sinais dos diferentes níveis de falhas em diferentes posições da estrutura, que são escolhidos aleatoriamente.

O módulo de monitoramento é composto por duas etapas, sendo a detecção e a classificação da falha. Os sinais em falhas são detectados através da avaliação e verificação do casamento com o conjunto de detectores próprios (sinais base-line), assim realiza-se a detecção de sinais em falha. Na sequência, ao identificar um sinal não-próprio (sinal com falha) realizase uma avaliação deste sinal, comparando-o com o conjunto de detectores de falhas, desta forma, ao ocorrer um casamento, o sinal é classificado com a classe do detector acionado. $\mathrm{O}$ monitoramento dos sinais é realizado de forma online.

O fluxograma do funcionamento do SMIE é apresentado na figura 3. 


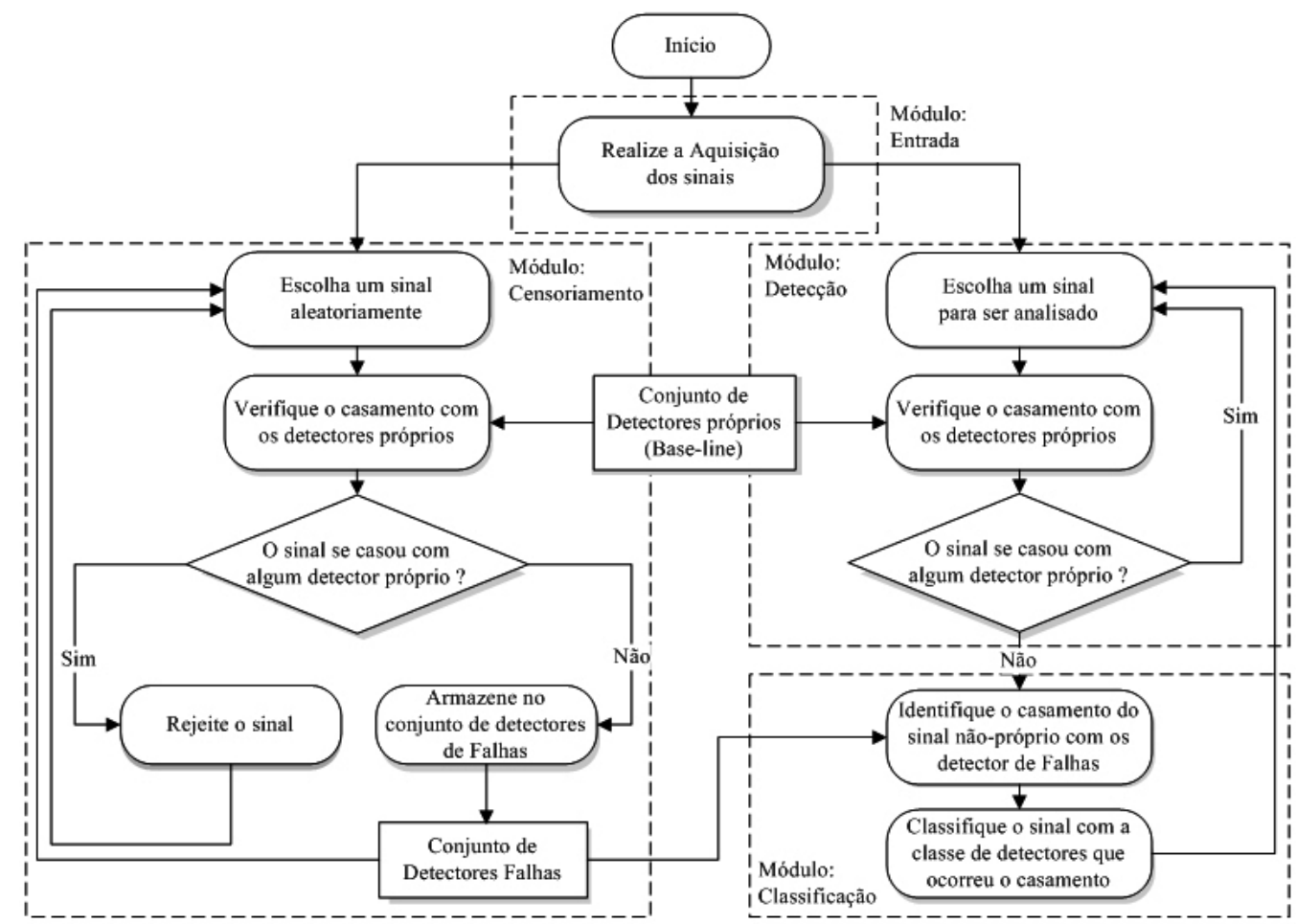

Figura 3: Fluxograma do SMIE.

\section{Aplicações e Resultados}

Nesta seção apresentam-se os resultados obtidos com a aplicação do SMIE na base de dados simulada. Todos os testes foram realizados utilizando um PC Intel Core 2 Duo $1.9 \mathrm{GHz}, 2 \mathrm{~GB}$ de Memória RAM, e sistema operacional Windows 7 Ultimate 32 bits. O algoritmo foi desenvolvido em MATLAB [11].

Nos testes realizados o objetivo foi avaliar a metodologia proposta, verificando a eficiência, precisão e o tempo computacional em relação a diferentes configurações do conjunto de detectores. Utilizou-se como parâmetro uma taxa com um valor fixo de 73,33\% (calculado com a equação (1)). Foram gerados três conjuntos de detectores $(I, I I$ e $I I I)$ utilizando $10 \%$, $20 \%$ e 30\% das amostras de cada padrão, totalizando 110, 220 e 330 detectores respectivamente. Sendo que para cada configuração cada padrão foi definido 1, 2 e 3 detectores respectivamente. A base de dados simulada conta com 110 padrões de falhas.

$\mathrm{Na}$ Tabela 2 apresentam-se os resultados obtidos pelo sistema de monitoramento da integridade de estruturas quando aplicado aos dados da viga de alumínio.

Tabela 2 - Resultados obtidos pelo método.

\begin{tabular}{cccc}
\hline \multirow{2}{*}{ Diagnóstico } & \multicolumn{3}{c}{ Conjunto de detectores } \\
\cline { 2 - 4 } & $I$ & $I I$ & III \\
\hline $\begin{array}{c}\text { Amostras } \\
\text { testadas }\end{array}$ & 1500 & 1500 & 1500 \\
\hline $\begin{array}{c}\text { Classificações } \\
\text { Corretas }\end{array}$ & 1482 & 1495 & 1500 \\
\hline $\begin{array}{c}\text { Classificações } \\
\text { Erradas }\end{array}$ & 18 & 5 & 0 \\
\hline Acerto (\%) & 98,88 & $99,66 \%$ & $100 \%$ \\
\hline Tempo (ms) & 160,5 & 163,4 & 162,8 \\
\hline
\end{tabular}

Cada teste foi executado 20 vezes a fim de garantir a veracidade dos resultados. Foi possível observar que o sistema de monitoramento da integridade de estruturas apresenta um bom desempenho (índice de acerto igual a $100 \%$ na melhor configuração), e que a quantidade de detectores influência diretamente no reconhecimento das falhas. Sugere-se utilizar 30\% das informações da base de dados para gerar o conjunto de detectores, visando proporcionar 
robustez ao sistema. Quanto mais conhecimento, mais eficiente é o processo de reconhecimento e classificação.

\section{Conclusão}

O SMIE proposto neste artigo apresentou excelentes resultados, obtendo um índice de acerto de $100 \%$ para a melhor configuração do sistema. A fase de geração de detectores é a que demanda maior tempo computacional, no entanto é executada de forma off-line não acarretando prejuízo ao algoritmo. A fase de monitoramento do sistema, a partir da aquisição dos sinais, é realizada rapidamente, com tempo inferior a 170 milésimos de segundo, o que credencia o SMIE a ser uma ferramenta utilizada em tempo real. Desta forma, conclui-se que o SMIE proposto com base nos sistemas imunológicos artificiais é bastante eficiente, confiável, robusto e preciso para realizar o monitoramento da integridade de estruturas aeronáuticas.

\section{Agradecimentos}

Os autores agradecem a CAPES e CNPQ (proc. $n^{\circ} 301769 / 2012-5$ ) pelo apoio financeiro de pesquisa.

\section{Referências}

[1] D. W. Bradley and A. M. Tyrrell. Immunotronics - Novel Finite-State-Machine Architectures with Built-In Self-Test Using Self-Nonself Differentiation. IEEE Transactions on Evolutionary Computation.Vol. 6, pp. 227-238 (2002).

[2] M. Chandrashekhar; R. Ganguli. Structural damage detection using modal curvature and fuzzy logic. Structural Health Monitoring, USA, v. 8, n. 4, p. 267-282, (2009).

[3] D. Dasgupta. "Artficial Immune Systems and Their Applications". Springer-Verlag New York, Inc., Secaucus, NJ, USA, 1998.

[4] L. N. de Castro. "Engenharia Imunológica: Desenvolvimento e Aplicação de Ferramentas Computacionais Inspiradas em Sistemas Imunológicos Artificiais". Tese de Doutorado, Faculdade de Engenharia Elétrica e de Computação, Universidade Estadual de Campinas, Campinas, Brasil, 2001.

[5] S. Forrest; A. Perelson; L. Allen and R. Cherukuri. Self-Nonself Discrimination in a computer, Proc. do IEEE Symposium on Research in Security and Privacy, pp. 202-212 (1994).

[6] V. R. Franco; D. D. Bueno; M. J. Brennan; A. A. Cavalini JR.; C. G. Gonsalez; V. Lopes JR. Experimental damage location in smart structures using Lamb waves approaches. In: Brazilian Conference on Dynamics, Control and Their Applications - DINCON. p. 1-4. (2009).

[7] V. Giurgiutiu. Tuned lamb wave excitation and detection with piezoelectric wafer active sensors for structural health monitoring. Journal of Intelligent Material Systems and Structures, USA, v. 16, n. 4, p. 291305, (2005).

[8] S. R. Hall. The effective management and use of structural health data. In: International Workshop on Structural Health Monitoring, p. 265-275. (1999).

[9] M. Krawczuk; W. Ostachowicz; G. Kawiecki. Detection of delaminations in cantilevered beams using soft computing methods. In: Conference on System Identification and Structural Health Monitoring, Madrid, p. $243-252$ (2000).

[10] F. P. A. Lima. "Analysis of Voltage Disturbances in Distribution Electrical Systems Based on Artificial Immune Systems”, Dissertation, University Estadual Paulista - UNESP, Ilha Solteira, March-2013, 169 p.

[11] Matlab (2011). 7.8 Version, Mathworks Company.

[12] A. Tebaldi; L. S. COELHO; V. LOPES JR. Detecção de falhas em estruturas inteligentes usando otimização por nuvem de partículas: fundamentos e estudo de casos. Revista Controle \& Automação, Brasil, v.17, n. 3, p. 312-330, 2006.

[13] S. Zheng; X. Wang; L. Liu. Damage detection in composite materials based upon the computational mechanics and neural networks. In: European Workshop on Structural Health Monitoring, Munich, p. 609-615 (2004).

[14] F. L. Wang; T. H. T. Chan; D. P. Thambiratnam and A. C. C. Tan. Damage Diagnosis for Complex Steel Truss Bridges Using Multi-Layer Genetic Algorithm. Journal of Civil structural Health Monitoring, SpringerVerlag, p. 117-217, (2013). 